\title{
Monitoring item and source information: Evidence for a negative generation effect in source memory
}

\author{
PAUL J. JURICA and ARTHUR P. SHIMAMURA \\ University of Califormia, Berkeley, California
}

\begin{abstract}
Item memory and source memory were assessed in a task that simulated a social conversation. Participants generated answers to questions or read statements presented by one of three sources (faces on a computer screen). Positive generation effects were observed for item memory. That is, participants remembered topics of conversation better if they were asked questions about the topics than if they simply read statements about topics. However, a negative generation effect occurred for source memory. That is, remembering the source of some information was disrupted if participants were required to answer questions pertaining to that information. These findings support the notion that item and source memory are mediated, as least in part, by different processes during encoding.
\end{abstract}

When one is engaged in conversation - say, at a social gathering or a business meeting - a variety of memory processes are operating. These processes facilitate the encoding of new information and the retrieval of relevant information from memory. At the same time, processing of source information can occur and can facilitate memory for such things as the individuals with whom one was conversing, the location of the conversation, and the time at which it occurred. What cognitive constraints limit our ability to encode and recollect these various aspects of memory? In particular, what encoding factors determine the degree to which one later recollects information about the content of a conversation, as opposed to information about the context in which a conversation took place?

Cognitive studies have revealed important contrasts between content memory and source memory. Many of these studies have distinguished memory for item information from memory for the contextual environment surrounding the learning episode (for a review, see Johnson, Hashtroudi, \& Lindsay, 1993). Conceptually, what distinguishes item memory from source memory is that item memory is less dependent on contextual information, whereas source memory depends critically on the acquisition of information within a spatiotemporal context. That is, source memory depends on autobiographical memory for the episode in which some information was learned. This distinction between item memory and source memory is exemplified in a variety of dichotomies, such as semantic versus episodic memory (Tulving, 1972, 1983), familiarity versus recollection (Jacoby, 1991; Jacoby \&

This research was supported by NIH Grants MH48757 and NS17778 to A.P.S. Correspondence concerning this article should be addressed to A. P. Shimamura, Department of Psychology, University of California, Berkeley, CA 94720-1650 (e-mail: aps@socrates.berkeley.edu).
Dallas, 1981), knowing versus remembering (Gardiner, 1988 ), and item versus context memory (Hirst, 1982; Mayes, Meudell, \& Pickering, 1985).

The source-monitoring framework, proposed by Johnson and colleagues (Johnson et al., 1993), provides a useful conceptualization for the analysis of item memory and source memory. In this framework, recollection is mediated by a variety of factors, including memory for perceptual information, memory for contextual information (i.e., spatial, temporal), memory for affective information, memory for semantic detail, and memory for the cognitive operations that were invoked during encoding. Source memory ability is determined largely by the extent to which these factors mark the context or episode in which information was presented. For example, memory for perceptual and contextual information appears to be critical for efficient source monitoring. In contrast, item memory may depend more on semantic detail and on cognitive operations that influence item strength or item familiarity. Thus, the source-monitoring framework can be employed to explain a variety of memory phenomena (see Conway \& Dewhurst, 1995; Hockley \& Cristi, 1996; Lindsay \& Johnson, 1991; Zaragoza \& Lane, 1994).

One factor that is known to strengthen the encoding of item information is the degree to which individuals engage in elaborative encoding. A powerful way to increase elaborative encoding is to require participants to selfgenerate information during learning. For example, Slamecka and Graf (1978) asked individuals to generate words in response to cues (e.g., RAPID-F___ ). Subsequent memory for words in this generate condition was significantly better than memory for words in a condition in which individuals simply read words for study (e.g., RAPID-FAST). The generation effect has been observed in a variety of test conditions, including free recall, cued recall, and recognition memory, and even in some implicit test conditions (Begg, Snider, Foley, \& Goddard, 1989; 
Gardiner, 1988; Glisky \& Rabinowitz, 1985; Graf, 1980; Hirshman \& Bjork, 1988; Jacoby, 1983; Slamecka \& Graf, 1978).

To what degree will the benefits of the self-generation of item information influence source memory performance? For item information, the generation effect appears to enhance the encoding and retention of item-specific information, relational information, and the cognitive operations involved in generation (Glisky \& Rabinowitz, 1985; Graf, 1980; Hirshman \& Bjork, 1988; McNamara \& Healy, 1995). This enhancement may extend to source memory, so that the generation of item information will lead to positive benefits for source memory. Specifically, an item-source enhancement hypothesis suggests that increases in item memory will be linked to enhancements of source memory. That is, a variable that influences memory encoding should generally affect item memory and source memory in the same direction. To the extent that the generation effect increases memory for item information, it should correspondingly boost source memory performance.

It may be, however, that item memory and source memory require somewhat different modes of processing. As a result, these different processing operations may compete for cognitive resources at the time of encoding. That is, there may be a tradeoff between the amount of processing allocated to item memory and the amount of processing allocated to source memory. Thus, an itemsource tradeoff hypothesis suggests that, in certain conditions, a positive effect for item memory would lead to a negative effect for source memory. This kind of tradeoff between item memory and source memory performance is consistent with the source-monitoring framework. For example, certain manipulations could promote encoding perceptual or contextual information at the sacrifice of increasing item strength; in such cases, source memory processing would benefit from this manipulation. Conversely, other manipulations may increase semantic detail or item strength at the expense of encoding perceptual or contextual information. Such findings would suggest that, in some circumstances, processing of item and source information will compete with each other.

Neuropsychological studies of brain-injured patients have suggested that item memory and source memory are dissociable from one another. Such findings provide biological evidence to suggest that, to some degree, item memory and source memory require different processing operations. For example, some but not all amnesic patients appear to exhibit an impairment in source memory that is out of proportion to their impairment in item memory (Schacter, Harbluk, \& McLachlan, 1984; Shimamura \& Squire, 1987, 1991). Further studies have suggested that the frontal lobes contribute significantly to source-monitoring performance. Patients with circumscribed frontal lobe lesions are not grossly amnesic and, thus, perform as well as control subjects on tests of fact or item memory. However, they exhibit disproportionate source-monitoring impairment (Janowsky, Shimamura, \& Squire, 1989; Shimamura, Janowsky, \& Squire, 1990). Finally, recent neuroimaging studies suggest increased frontal lobe involvement during tasks that require source monitoring or episodic memory retrieval (Petrides, Alivisatos, \& Evans, 1995; Tulving, Kapur, Craik, Moscovitch, \& Houle, 1994).

In the present study, we assessed the effects of selfgenerating item information on both item memory and source memory. To portray an everyday setting, we engaged participants in simulated social conversations by having them "interact" with three computer-generated individuals (i.e., faces on a computer screen). During a study phase, the participants generated answers in response to questions presented by one of the three individuals (e.g., "What type of sports do you like to watch?"). In addition, the participants simply read statements presented by one of the three individuals (e.g., "I think that dogs make great pets"). Item memory was tested by asking the participants to recall the conversational topics presented during the study phase. Source memory was tested by asking the participants to identify the individual who presented a study item (see Figure 1).

A generation effect for item memory in this paradigm would be reflected in better memory for topics presented as questions, rather than as statements. That is, memory for topics is expected to be enhanced when the participants are required to generate an answer to a question related to a topic. If the generation effect extends to the encoding of source information, source memory for items presented as questions would also be enhanced (itemsource enhancement hypothesis). If, however, there is a tradeoff between the processing of item memory and source memory (item-source tradeoff hypothesis), a facilitation in item memory owing to the generation effect would lead to decrements in source memory performance for those items. That is, the item-source tradeoff hypothesis predicts that a positive generation effect for item memory will lead to a negative generation effect for source memory.

\section{EXPERIMENT 1}

\section{Method}

Participants. Sixteen undergraduate students from the University of California at Berkeley participated in this experiment in exchange for partial fulfillment of a course requirement. They averaged 19.2 years of age and were native English speakers.

Apparatus and Design. An Apple Macintosh computer was used to present stimuli and record responses. The stimuli consisted of computer-generated line drawings of male faces (see Figure 1) that were inserted into a HyperCard program (Apple Computer, Version 2.0). In the study phase, one of the three faces (i.e., the sources) was associated with a set of items. There were a total of 24 items, each of which could be presented as a question (e.g., "What type of music do you like to listen to?"; "What is your primary means of transportation?") or as a statement (e.g., "The type of music I like to listen to is jazz"; "My primary means of transportation is my bike"). For each participant, 12 items were presented as 

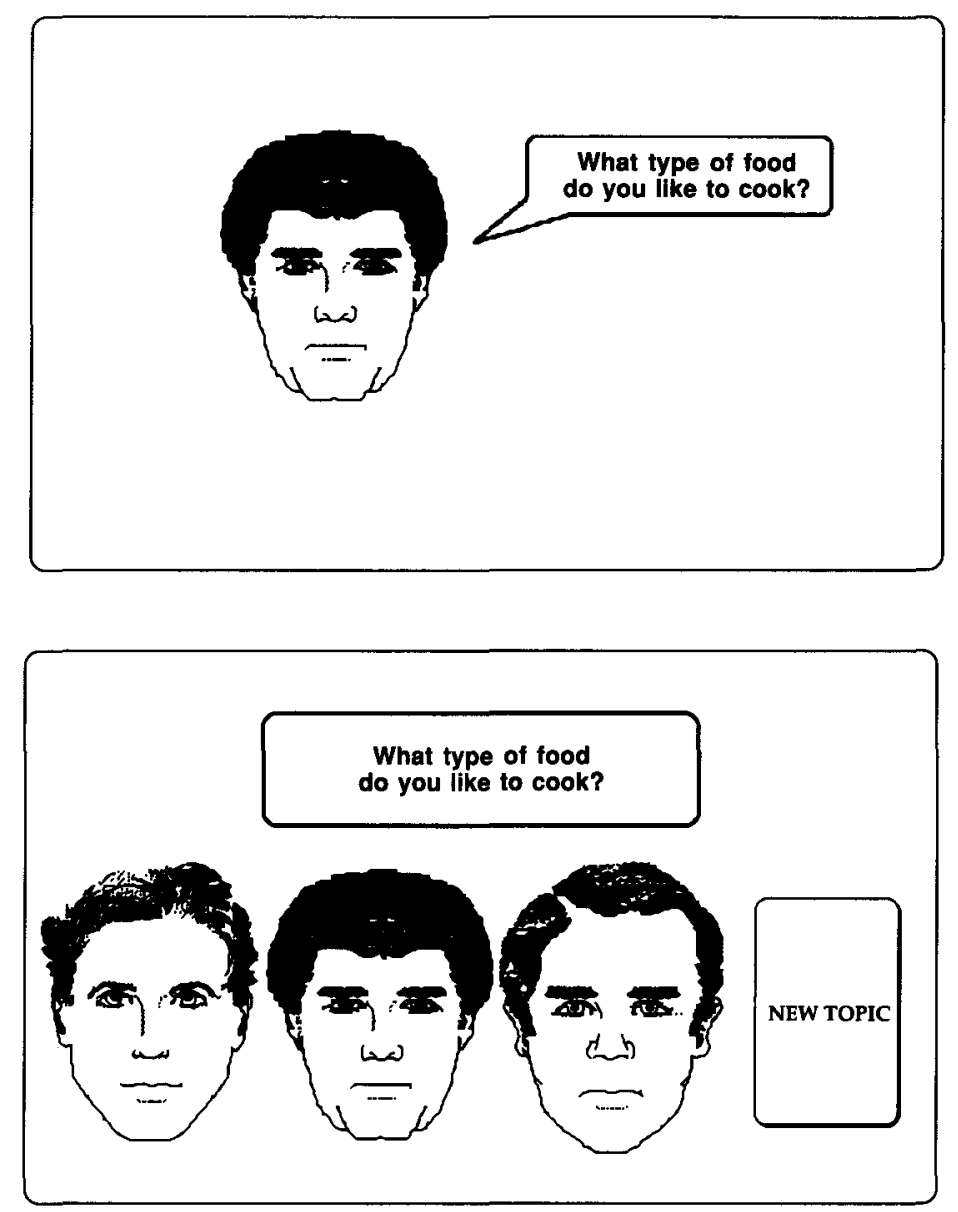

Figure 1. During the study phase, the participants were shown statements or questions presented by one of three faces (top display). During the source recognition phase, the participants were asked to identify which of the three faces presented a study item or whether the item was a new one not previously presented during the study phase (bottom display).

questions, and the other 12 were presented as statements. Across participants, the items were counterbalanced across the two presentation formats. Each source presented four questions and four statements.

The topics presented in the statements and questions were meant to mimic the kind of information exchanged during social conversations. In particular, the statements and questions referred to personal tastes and interests. Topics covered information on such issues as food preferences, geography, family, sports, and entertainment.

Procedure. In the study phase, questions and statements were presented in six blocks, each consisting of four trials (two statements and two questions) presented by a particular source. Across the entire study phase, two blocks of trials were presented by each source, with the restriction that the same source would never appear in consecutive blocks. Otherwise, the order of presentation was random. The participants were instructed to try to remember the items and to associate the items with the sources. For statements, they were instructed to read the statement to themselves, but no verbal response was required. For questions, they were instructed to read the question to themselves and to generate an answer to the question. The experimenter entered the answer into the computer. The items appeared on the screen for $7 \mathrm{sec}$. For question items, if a participant did not answer a question within $7 \mathrm{sec}$, a cue appeared that indicated that a response was required. With this method, an answer for each question was elicited from all the participants.

Following the study phase, a free recall test was given for all of the topics presented during study. For this test, the participants were instructed to report as many topics as they could remember from both questions and statements. They could list the items in any order and were not required to specify who presented the item or whether the item was presented as a statement or as a question. On this test, a correct recall score required the participant to identify the topic rather specifically. That is, the response had to indicate a specific fact (e.g., "there was a question about riding a bike") rather than a more general recollection (e.g., "there was a question about transportation or vehicles"). Memory for the topics could be gleaned from the stimulus information (presented as statements or as questions) and thus did not depend on the participants' responses. The participants were given $3 \mathrm{~min}$ to recall the topics.

Following the item recall test, a source recognition test was given for the 24 study items and 6 new items (three new statements and three new questions). The 6 new items were included in order to assess the ability to discriminate new items from study items. For 


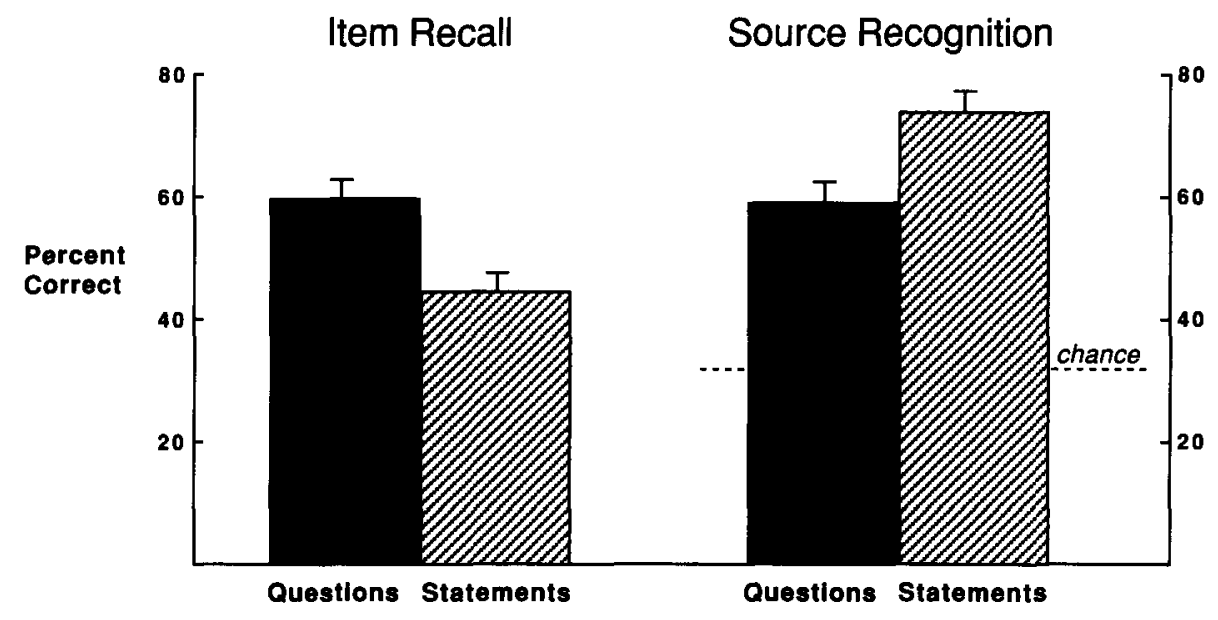

Figure 2. Item recall and source recognition performance for items presented as questions or statements (Experiment 1). Note the positive generation effect for item memory and the negative generation effect for source memory.

each trial, an item (statement or question) was shown in the presence of the three sources (see Figure 1). In addition, a fourth choice (new item) was to be chosen if the participant judged an item to be one that was not presented in the study phase. The responses were made with a mouse device by having the participants point and select one of the four choices. The source test was self-paced, and the order for the 24 old and 6 new items was randomized.

\section{Results and Discussion}

Figure 2 displays item recall and source recognition memory performance for items presented as statements and questions. On the item recall test, the participants recalled significantly more topics from questions $(59.4 \%)$ than from statements $[42.7 \% ; t(15)=3.81, p<.01]$. These findings indicate that the act of generating answers to questions facilitated item recall. Thus, the findings support a generation effect for item recall in this paradigm.

On the source recognition test, the participants were very accurate at recognizing the new items-that is, items that had never appeared in the study phase. In fact, none of the participants falsely identified a new item as a study item. Also, the participants exhibited a low rate of recognition failure for studied items - that is, falsely identifying a studied item as a new item. Specifically, only $1.0 \%$ of the studied questions and $4.2 \%$ of the studied statements were incorrectly identified as new. The difference in recognition failure between questions and statements was not statistically significant $(t=1.7, p=$ $.10)$. Thus, the participants were able to discriminate old items from new items.

To assess source memory performance, we analyzed those items that participants identified as having been presented by one of the three sources. That is, we did not include the few items that were falsely identified as a new. As a result, source recognition performance was based on a chance level of $33 \%$. Other studies have used simi- lar measures to analyze source forgetting (e.g., Craik, L. W. Morris, R. G. Morris, \& Loewen, 1990; Schacter, Osowiecki, Kaszniak, Kihlstrom, \& Valdiserri, 1994). Source memory performance was significantly poorer for items presented as questions $(58.2 \%)$ than for items presented as statements $[76.5 \% ; t(16)=4.9, p<.01]$. Thus, a negative generation effect was observed for source memory. That is, generating an answer to a question reduced the probability of remembering source information.

In this study, our intent was to bring the item-source paradigm into a more ecologically significant domain, such as the simulation of a social conversation. On the basis of this goal, participants either responded verbally to questions presented on a computer screen or silently read statements. Thus, there was a somewhat different requirement for each presentation format (question vs. statement). Specifically, we did not require verbal responses for items presented as statements. It is reasonable that this difference may have boosted the positive generation effect observed for items presented as questions. It is less obvious how this difference could account for the decrease in source memory for questions. Presumably, the encoding or focusing of item information, as opposed to the mere verbalization of information, reduced the ability to adequately acquire source information.

In sum, generating answers to questions increased item memory, as compared with reading statements. Interestingly, this positive generation effect was limited to item memory. On a test of source memory, a negative generation effect occurred, so that the act of generating answers to questions reduced memory for the face (source) that was associated with that item. Although direct comparisons between two different dependent measures must be viewed with caution, a two-way analysis of variance (ANOVA), with study item (questions vs. statements) and test measure (item recall vs. source recognition) as factors, 


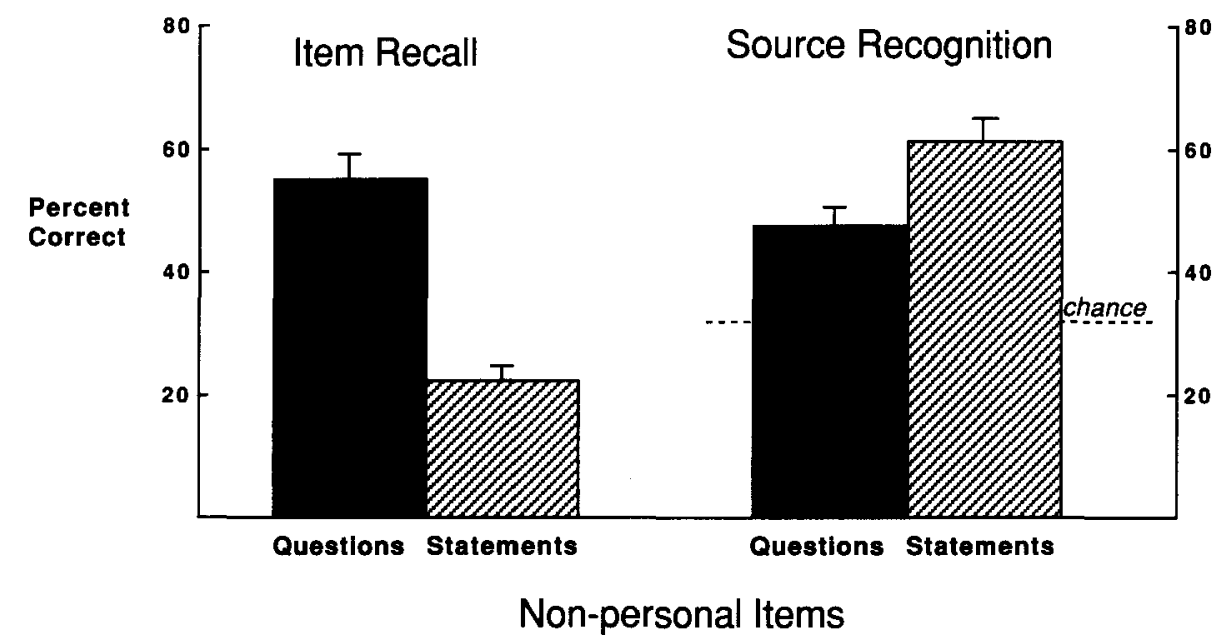

Figure 3. Item recall and source recognition performance for nonpersonal items presented as questions or statements (Experiment 2).

revealed a significant cross-over interaction $[F(15,1)=$ 47.4, $\left.M S_{\mathrm{e}}=4,878, p<.01\right]$. These findings confirm a tradeoff between the processing of item information and the processing of source information during encoding.

\section{EXPERIMENT 2}

In Experiment 1, a tradeoff between item memory and source memory was observed for person-oriented topics, such as individual habits, tastes, likes, and dislikes (e.g., "What type of sports do you like to watch?"; "I think that dogs make great pets"). Although this set of items provided a simulation close to discourse during conversations, it is possible that source recognition for statements was better than that for questions because questions referred to the participant's preferences, whereas statements referred to the source's preferences. In Experiment 2, we assessed item memory and source memory for depersonalized questions and statements. We used the same paradigm as that in the first experiment, but modified the study items by making them nonpersonal (e.g., "What type of sports are commonly watched on television?"; "Many people think that dogs make great pets"). Such items refer to general preferences and are not inherently biased toward either the source's or the participant's preferences. If the item-source tradeoff hypothesis is correct, the same pattern of results should be observed for nonpersonal items as was observed for items used in Experiment 1 . Specifically, we predict a positive generation effect for item recall and a negative generation effect for source memory.

\footnotetext{
Method

Participants. Twenty undergraduate students at the University of California at Berkeley, who volunteered in exchange for partial fulfillment of a course requirement, participated in the experiment. They averaged 20.1 years of age and were native English speakers.

Stimuli. The statements and questions used in this experiment were depersonalized versions of those used in Experiment 1. Instead of using personal pronouns (e.g., " $M y$ primary means of trans-
}

portation is my bicycle"; "Where do you think the most scenic spot in California is located?"), the participants studied nonpersonal questions and statements (e.g., "Bicycles are a common means of transportation these days"; "What is a very scenic spot in California?"). Thus, statements and questions were not inherently biased toward the sources or the participants.

Procedure. The only change in this experiment was the use of nonpersonal items. The apparatus and test procedure were identical to those used in Experiment 1. In summary, the participants either read statements or provided answers to questions. The 24 items ( 12 statements, 12 questions) were presented by one of three computergenerated faces. On the item recall test, the participants were asked to report as many topics of conversation that were discussed during the study phase as they could recall. On the source recognition test, the 24 old items and 6 new ones were shown in the presence of the three faces. The participants were asked to determine which of the three faces presented the item or whether the item was a new one.

\section{Results and Discussion}

As in Experiment 1, the participants were able to discriminate new items from study items. Only 1 participant made a false alarm error by indicating that a new item was presented at study. Figure 3 displays item recall and source recognition memory performance for the nonpersonal items. The findings replicated the findings of Experiment 1 . On the item recall test, the participants recalled more topics from questions $(55.4 \%)$ than from statements $[22.1 \% ; t(19)=6.89, p<.01]$. Yet, on the source recognition test, the participants recognized the source of items presented as statements $(60.3 \%)$ better than they were able to recognize the source of items presented as questions $[47.9 \% ; t(19)=2.25, p<.05]$. A two-way ANOVA, with study item (questions vs. statements) and test measure (item recall vs. source recognition) as factors, revealed a significant cross-over interaction $\left[F(1,19)=27.6, M S_{\mathrm{e}}=1,047, p<.01\right]$.

These findings of memory performance for nonpersonal items replicated those obtained in Experiment 1. Thus, biases in the use of personalized statements and questions cannot account for the finding of a positive generation effect for item memory and a negative generation 


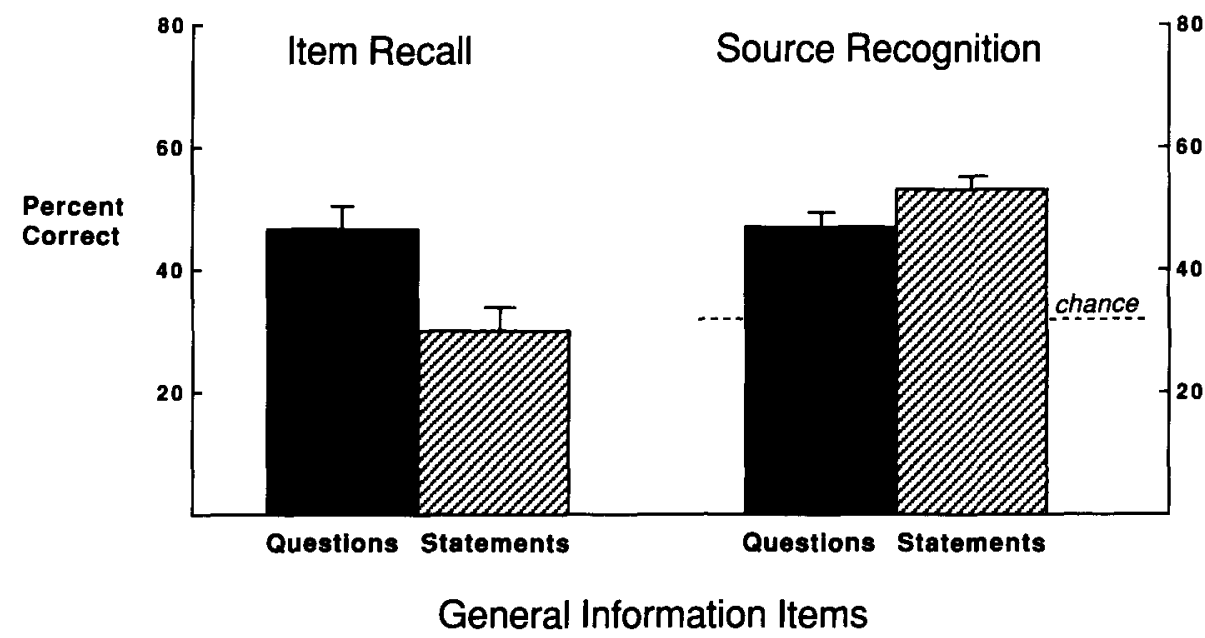

Figure 4. Item recall and source recognition performance for trivia items presented as questions or statements (Experiment 3).

effect for source memory. This tradeoff between item memory and source memory supports the item-source tradeoff hypothesis of memory acquisition.

\section{EXPERIMENT 3}

In a third experiment, we investigated memory for the kind of items used in previous studies of fact learning and source memory. In previous studies (e.g., Schacter et al., 1984; Shimamura \& Squire, 1987), participants were presented fictitious or general knowledge facts. For example, Shimamura and Squire (1987) asked participants to learn trivia facts, such as "The name of the dog on the Cracker Jacks box is Bingo," and later tested item memory and source memory. In the present study, we assessed generation effects by having three different sources present general knowledge in the form of statements ("The name of Tarzan's girlfriend is Jane") and questions ("What is the name of the comic strip character who eats spinach to increase his strength?"). This kind of information is purely factual in nature and, thus, would not have any explicit or implicit association with either the participants or the sources. To the extent that the itemsource tradeoff hypothesis can be generalized to these kinds of facts, we predicted a positive generation effect for item recall and a negative generation effect for source recognition.

\section{Method}

Participants. Thirty-two participants, who volunteered in exchange for partial fulfillment of a course requirement, participated in this experiment. They averaged 18.7 years of age and were native English speakers.

Stimuli. General knowledge information was presented as statements or questions. The items came from the set of general information questions developed and normed by Nelson and Narens (1980). The facts chosen for this experiment were those that were frequently answered in the normative study (FREQUENCY RANK). All the items could be presented as statements or as questions. For a given participant, half of the items were presented as statements (e.g., "Benjamin Franklin is the man who showed that lightning is electricity"; "A surgeon is a medical doctor who specializes in cutting the body"), and half were presented as questions ("What is the name of the person who showed that lightning is electricity?"; "What is the name of a medical doctor who specializes in cutting the body?").

Procedure. The only change in this experiment was the use of general information (i.e., trivia) items. The apparatus and test procedure were identical to those used in the previous two experiments. In summary, the participants either read statements or provided answers to questions. The 24 items ( 12 statements, 12 questions) were presented by one of three computer-generated faces. On the item recall test, the participants were asked to report as many topics of conversation that were discussed during the study phase as they could recall. On the source recognition test, the 24 old items and 6 new ones were shown in the presence of the three faces. The participants were asked to determine which of the three faces presented the item or whether the item was a new one.

\section{Results and Discussion}

Figure 4 displays item recall and source recognition memory performance for general knowledge. The findings replicated those obtained in Experiments 1 and 2. On the item memory test, the participants recalled significantly more topics from questions $(47.1 \%)$ than from statements $[29.4 \% ; t(31)=5.11, p<.01]$. On the source recognition test, the participants were better able to recognize the source of items presented as statements (53.7\%) than that of items presented as questions $[47.4 \% ; t(31)=$ $2.19, p<.05]$. Moreover, a two-way ANOVA with study item (questions vs. statements) and test measure (item recall vs. source recognition) revealed a significant crossover interaction $\left[F(31,1)=31.1, M S_{\mathrm{e}}=3,980, p<.01\right]$. Compared with the previous two experiments, the advantage of statements over questions on the source memory test appeared to be less strong, although still statistically significant.

These findings provided a third replication of a positive generation effect for item recall and a negative gen- 
eration effect for source memory. The findings also converge with previous studies of fact learning and source memory by using the same kinds of general information items used previously (Shimamura \& Squire, 1987).

\section{GENERAL DISCUSSION}

In three experiments, we assessed item memory and source memory in an experimental paradigm that simulated a social conversation. In this paradigm, individuals were shown one of three computer-generated faces (sources) and were asked either to generate answers to questions or to read statements given by the sources. In these experiments, a positive generation effect was observed on tests of item memory, whereas a negative generation effect was observed on tests of source memory. Specifically, memory for the topics of conversation was better recalled for items presented as questions than for items presented as statements. However, the reverse was true for source memory-that is, memory of the person who presented a topic of conversation was better for items presented as statements than for items presented as questions. These findings support an item-source tradeoff hypothesis in which the encoding of item and source information are, to some extent, dissociable from one another.

These findings indicate that memory for an episode is not a unified phenomena. If memory for items and memory for source were inherently unified, a variable that increased item memory, such as elaborative encoding, should have correspondingly benefited source memory. However, in the present investigation, source memory was reduced by a variable that increased item memory. This tradeoff in memory processing was observed for several kinds of information, such as personal tastes and preferences (Experiment 1), generic preferences (Experiment 2), and even general information knowledge, which is purely semantic and remote from contextual information (Experiment 3 ). Thus, the tradeoff in the encoding of content and contextual knowledge is a rather robust phenomenon.

Related findings have been observed in other studies of source monitoring. For example, Lindsay and Johnson (1991) presented word pairs and asked individuals either to form a sentence using both words (deep-deep condition) or to form a sentence using the left word and to perform a shallow task on the right word (deep-shallow condition). An item-source tradeoff effect was observed: Item recognition for words presented on the right was better in the deep-deep condition than in the deepshallow condition, whereas source memory performance (i.e., determining whether a word was presented on the left or the right) was better in the deep-shallow condition than in the deep-deep condition. In a study of eyewitness memory, Zaragoza and Lane (1994) embedded misleading information in either questions or narratives that the individuals read after observing a slide show depicting a theft. Source errors were greater when misleading information was embedded in questions than when it was embedded in narratives. However, in this study, item memory was not different between the two conditions.
The present findings are consistent with interpretations based on the source-monitoring framework developed by Johnson and colleagues (Johnson et al., 1993). That is, item memory and source memory form memory representations that are based on a variety of attributes, including perceptual, contextual, semantic, affective, and operational attributes. Performance on item memory and source memory tests will be dependent on the extent to which these attributes are processed. In our study, the generation effect can be assumed to increase semantic detail or item familiarity. Thus, item memory was better for questions than for statements because items presented as questions were benefited by the generation of an answer. Interestingly, this benefit in item memory was associated with a decrement in source memory. Although the sourcemonitoring framework does not necessarily predict a tradeoff between item and source processing, the framework does suggest the possibility, since it is assumed that item memory and source memory depend, in part, on different encoding processes. Apparently, in our study, the act of generating answers to questions not only facilitated item memory, but also reduced processing of perceptual and contextual information important for source memory.

The finding of generation effects on tasks involving the recollection of factual information extends the conditions under which generation effects can be observed. Previous studies have observed a benefit for the generation of single words (Glisky \& Rabinowitz, 1985), paired associates (Begg et al., 1989; Hirshman \& Bjork, 1988; Slamecka \& Graf, 1978), arithmetic calculations (McNamara \& Healy, 1995), and performed actions (Kausler, Lichty, \& Freund, 1985). In the present study, generating answers to general information questions improved memory for the topics associated with factual questions.

To the extent that the kind of generation effects observed in our investigation is similar to those observed in other studies, it is possible to study the item-source tradeoff hypothesis in more traditional paradigms. Indeed, Jurica (1997) asked participants to read word pairs (TEST-HEAT) or to generate word fragments using letters from a word cue (TESI-H_A_). The cue words were semantically related (RAGE-HATE) or unrelated (TEST-HEAT) to the target words. A free recall test was then given to assess memory for all the words presented. As would be expected on the basis of the generation effect, target words generated by fragments were remembered better than target words that were read (related findings were reported by McDaniel \& Waddill, 1990). However, in the unrelated condition, a negative generation effect was observed for cue words. That is, memory for unrelated cue words (the word TEST in the example above) was poorer when it was presented with a word fragment (generate condition) than when it was presented with an intact word (read condition).

The negative generation effect observed for unrelated cue words in Jurica (1997) has similarities with the negative generation observed in the present study. In both cases, the negative effects were observed when to-be- 
remembered information was arbitrarily associated with target information. That is, for both unrelated word pairings and item-source (face) pairings, the associations were random. Apparently, processing or attentional tradeoffs are significant when unrelated information is presented within the same episodic context. In Jurica, positive generation effects were observed for related cue words. In this condition, the associative link between related cue words and target words apparently strengthens the relationship between these two words when they are presented within the same episodic context. Thus, the positive generation effect for fragment target words led to a carryover effect for related cue words.

In source memory experiments, the finding of tradeoffs between item memory and source memory suggests that the encoding of item information can be quite distinct from the encoding of the spatiotemporal context in which the information is presented. Related findings have been observed in other memory tasks, such as those used to assess encoding specificity, levels of processing, or transfer-appropriate processing (Morris, Bransford, \& Franks, 1977; Roediger, 1990). To what extent is the itemsource distinction different from any other distinction between two aspects of a memory representation? It may be that source memory does not have any special status, except that it depends significantly on associative memory between an item and some aspect of the spatiotemporal context in which the item was learned. On this view, the distinction between item memory and source memory is related to the distinction between item and interitem (i.e., relational) memory (Anderson \& Bower, 1972; Mandler, 1980).

Tradeoffs between the encoding of item and relational memory have been shown to occur (Anderson \& Bower, 1972; Begg, 1979; Hockley \& Cristi, 1996; Hunt \& Einstein, 1981) and appear to be similar to the tradeoff of item and source information. For example, in some cases, encoding processes that emphasize item information reduce encoding of relational memory (Hockley \& Cristi, 1996). Interestingly, encoding processes that emphasize relational information do not tend to reduce item memory. This kind of asymmetric tradeoff is reminiscent of the item-source tradeoff observed in Zaragoza and Lane (1994). Thus, on this view, source memory is a kind of associative or relational memory-one that ties together item information and contextual information. Of course, this kind of association has some special status, as it is the basis for episodic memory, the kind of memory that is a hallmark of our ability to recollect the past.

One factor that appears to have an effect on the magnitude of the item-source tradeoff effect is the degree to which processing is directed to the source or to the items. That is, the tradeoff effect could have been mediated by the fact that questions pertained to individuals and items pertained to sources. For example, in Johnson, Nolde, and De Leonardis (1996), individuals heard statements and were asked to focus on how they felt about the statement (selffocus) or how they thought the speaker felt about the statement (other-focus). The self-focus condition produced equal or better item recognition-but poorer source recognition-as compared with the other-focus condition. Similar findings were obtained in a remember/know paradigm in which self-reference increased the number of remembered judgments (Conway \& Dewhurst, 1995). Self-focusing or self-referencing could have accentuated the tradeoff effect in Experiment 1, because questions were asked about the participants' personal interests, whereas statements pertained to the sources' interests. This effect, however, cannot adequately account for the findings of Experiments 2 and 3 because, in those experiments, the items were not directed to either individuals or sources and, nevertheless, item-source tradeoff effects were observed.

To some extent, the item-source tradeoffs observed in the present investigation are reminiscent of previous analyses involving resource allocation and selective attention (Baddeley, 1986; Kahneman, 1973; Norman \& Shallice, 1986). That is, the findings could be interpreted as instances in which task demands influence working memory or executive control of information processing. This kind of control would determine the amount of processing to be allocated to aspects of an episode and, thus, determine ultimately what is stored in long-term memory. This rather interesting relation suggests that item and source information - two inherent aspects of episodic memory - are distinct enough that increases in the processing of one can sometimes deter processing of the other. These findings may thus provide insights into the processing limitations in working memory and the manner in which new episodic representations are established.

Interestingly, executive control, working memory, and source monitoring all have been associated with frontal lobe function (Baddeley, 1986; Moscovitch, 1994; Shimamura, 1995). As such, the present findings suggest that problems in working memory and resource allocation may account for source memory impairment observed in patients with frontal lobe lesions (Janowsky et al., 1989). Specifically, patients with frontal lobe lesions may exhibit problems in source memory because they fail to allocate enough cognitive resources to the processing of source information, perhaps because control processes are aberrant or because of limited cognitive resources. On the basis of this view, patients with frontal lobe lesions do not exhibit deficits in source memory per se but, rather, have a general problem in organizing and executing cognitive control in many domains (see Moscovitch, 1994; Shimamura, 1996). This interpretation is consistent with other findings of frontal lobe dysfunction-for example, increased susceptibility to proactive interference and deficits in a variety of tasks, such as inference making, metamemory, memory for temporal order, and memory retrieval (Janowsky et al., 1989; Petrides, 1989; Shimamura, 1996; Shimamura et al., 1990). 


\section{REFERENCES}

ANDERSON, J. R., \& Bower, G. H. (1972). Recognition and retrieval processes in free recall. Psychological Review, 79, 97-123.

BADDELEY, A. (1986). Working memory. Oxford: Oxford University Press.

BEGG, I. (1979). Trace loss and the recognition failure of unrecalled words. Memory \& Cognition, 7, 113-123.

BEGG, I., SNIDER, A., FolEy, F., \& GodDARD, R. (1989). The generation effect is no artifact: Generating makes words distinctive. Journal of Experimental Psychology: Learning, Memory, \& Cognition, 15, 977 989.

Conway, M. A., \& Dewhurst, S. A. (1995). Remembering, familiarity, and source monitoring. Quarterly Journal of Experimental Psychology, 48A, 125-140.

Craik, F. I. M., Morris, L. W., Morris, R. G., \& LoEwen, E. R. (1990) Relations between source amnesia and frontal lobe functioning in older adults. Psychology \& Aging, 5, 148-151.

GaRDINER, J. M. (1988). Generation and priming effects in wordfragment completion. Journal of Experimental Psychology: Learning, Memory, \& Cognition, 14, 495-501.

GliskY, E. L., \& RABINOWITZ, J. C. (1985). Enhancing the generation effect through repetition of operations. Journal of Experimental Psychology: Learning, Memory, \& Cognition, 11, 193-205.

GRAF, P. (1980). Two consequences of generating: Increased inter- and intraword organization of sentences. Journal of Verbal Learning \& Verbal Behavior, 19, 316-327.

Hirshman, E., \& BJork, R. A. (1988). The generation effect: Support for a two-factor theory. Journal of Experimental Psychology: Learning, Memory, \& Cognition, 14, 484-494.

HIRST, W. (1982). The amnesic syndrome: Descriptions and explanations. Psychological Bulletin, 91, 435-460.

HOCKLEY, W. E., \& CRISTI, C. (1996). Tests of the separate retrieval of item and associative information using a frequency-judgment task. Memory \& Cognition, 24, 796-811.

Hunt, R., \& EINSTEIN, G. O. (1981). Relational and item-specific information in memory. Journal of Verbal Learning \& Verbal Behavior, 20, 497-514.

JACOBY, L. L. (1983). Perceptual enhancement: Persistent effects of an experience. Journal of Experimental Psychology: Learning, Memory, \& Cognition, 9, 21-38.

JACOBY, L. L. (1991). A process dissociation framework: Separating automatic from intentional uses of memory. Journal of Memory \& Language, 30, 513-541.

JACOBY, L. L., \& DALLAS, M. (1981). On the relationship between autobiographical memory and perceptual learning. Journal of Experimental Psychology: General, 110, 306-340.

Janowsky, J. S., Shimamura, A. P., \& Squire, L. R. (1989). Source memory impairment in patients with frontal lobe lesions. Neuropsychologia, 27, 1043-1056.

Johnson, M. K., Hashtroudi, S., \& Lindsay, D. S. (1993). Source monitoring. Psychological Bulletin, 114, 3-28.

Johnson, M. K., Nolde, S. F., \& De Leonardis, D. M. (1996). Emotional focus and source monitoring. Journal of Memory \& Language, $35,135-156$.

JURICA, P. J. (1997). A cognitive and neuropsychological analysis of generation effects. Unpublished doctoral dissertation, University of California, Berkeley.

Kahneman, D. (1973). Attention and effort. Englewood Cliffs, NJ: Prentice-Hall.

Kausler, D. H., Lichty, W., \& Freund, J. S. (1985). Adult age difference in recognition memory and frequency judgments for planned versus performed activities. Developmental Psychology, 21, 647654.

LiNDSAY, D. S., \& JoHnSON, M. K. (1991). Recognition memory and source monitoring. Bulletin of the Psychonomic Society, 29, 203-205.

MANDLER, G. (1980). Recognizing: The judgment of previous occurrence. Psychological Review, 87, 252-271.

Mayes, A. R., Meudell, P. R., \& Pickering, A. (1985). Is organic am- nesia caused by a selective deficit in remembering contextual information? Cortex, 21, 167-202.

MCDANIEL, M. A., \& WADDILL, P. J. (1990). Generation effects for context words: Implications for item-specific and multifactor theories. Journal of Memory \& Language, 29, 201-211.

MCNAMARA, D. S., \& HealY, A. F. (1995). A procedural explanation of the generation effect: The use of an operand retrieval strategy for multiplication and addition problems. Journal of Memory \& Language, 34, 399-416.

MorRIS, C. D., Bransford, J. D., \& FranKs, J. J. (1977). Levels of processing versus transfer appropriate processing. Journal of Verbal Learning \& Verbal Behavior, 16, 519-533.

Moscovirch, M. (1994). Cognitive resources and dual-task interference effects at retrieval in normal people: The role of the frontal lobes and medial temporal cortex. Neuropsychology, 8, 524-534.

NELSON, T. O., \& NARENS, L. (1980). A new technique for investigating the feeling of knowing. Acta Psychologica, 46, 69-80.

Norman, D. A., \& Shallice, T. (1986). Attention to action: Willed and automatic control of behavior. In R. J. Davidson, G. E. Schwartz, \& D. Shapiro (Eds.), Consciousness and self-regulation (pp. 1-18). New York: Plenum.

Petrides, M. (1989). Frontal lobes and memory. In F. Boller and J. Grafman (Eds.), Handbook of neuropsychology (Vol. 3, pp. 75-90). Amsterdam: Elsevier.

Petrides, M., Alivisatos, B., \& Evans, A. C. (1995). Functional activation of the human ventrolateral frontal cortex during mnemonic retrieval of verbal information. Annals of the New York Academy of Sciences, 92, 5803-5807.

ROEDIGER, H. L., III (1990). Implicit memory: Retention without remembering. American Psychologist, 45, 1043-1056.

Schacter, D. L., Harbluk, J., \& McLachlan, D. (1984). Retrieval without recollection: An experimental analysis of source amnesia. Journal of Verbal Learning \& Verbal Behavior, 23, 593-611.

Schacter, D. L., Osowiecki, D., KaszNiaK, A. W., Kihlstrom, J. F., \& VALDISERRI, M. (1994). Source memory: Extending the boundaries of age-related deficits. Psychology \& Aging, 9, 81-89.

Shimamura, A. P. (1995). Memory and frontal lobe function. In M. S. Gazzaniga (Ed.), The cognitive neurosciences (pp. 803-813). Cambridge, MA: MIT Press.

SHIMAMURA, A. P. (1996). The control and monitoring of memory functions. In L. Reder (Ed.), Metacognition and implicit memory (pp. 259274). Mahwah, NJ: Erlbaum.

Shimamura, A. P., Janowsky, J. S., \& SQuire, L. R. (1990). Memory for the temporal order of events in patients with frontal lobe lesions and amnesic patients. Neuropsychologia, 28, 803-813.

Shimamura, A. P., \& SQuire, L. R. (1987). A neuropsychological study of fact memory and source amnesia. Journal of Experimental Psychology: Learning, Memory, \& Cognition, 13, 464-473.

Shimamura, A. P., \& SQuire, L. R. (1991). The relationship between fact and source memory: Findings from amnesic patients and normal subjects. Psychobiology, 19, 1-10.

Slamecka, N. J., \& GraF, P. (1978). The generation effect: Delineation of a phenomenon. Journal of Experimental Psychology: Human Learning \& Memory, 4, 592-604.

Tulving, E. (1972). Episodic and semantic memory. In E. Tulving \& W. Donaldson (Eds.), Organization of memory (pp. 381-403). New York: Academic Press.

Tulving, E. (1983). Elements of episodic memory. Oxford: Oxford University Press, Clarendon Press.

Tulving, E., Kapur, E. S., Craik, F. I. M., Moscovitch, M., \& Houle, S. (1994). Hemispheric encoding/retrieval asymmetry in episodic memory: Positron emission tomography finding. Proceedings of the National Academy of Sciences, 91, 2016-2020.

ZARAGOZA, M. S., \& LANE, S. M. (1994). Source misattributions and the suggestibility of eyewitness memory. Journal of Experimental Psychology: Learning, Memory, \& Cognition, 20, 934-945.

(Manuscript received June 2, 1997; revision accepted for publication July 21,1998 .) 